Canad. Math. Bull. Vol. 22 (2), 1979

\title{
INTERNAL HOM FUNCTORS FOR POLARITIES
}

\author{
BY \\ EVELYN NELSON
}

The notion of functionality for an internal hom functor $H$ in a concrete category $\mathbf{K}$ was introduced in Banaschewski and Nelson [1], formalizing the condition that the structure on the $H(A, B)$ is "pointwise" structure on sets of functions. It was proved there (Proposition 3) that, for a functor $\otimes: \mathbf{K} \times \mathbf{K} \rightarrow$ $\mathbf{K}$ and an internal hom $H$ on $\mathbf{K}$, any two of the following conditions implied the third: $H$ is functional, $\otimes$ is the functor of universal bimorphisms, and $\otimes$ is a tensor multiplication for $H$, where the latter means that there is a natural equivalence $\mathbf{K}(A \otimes B, C) \cong \mathbf{K}(A, \grave{H}(B, C))$.

This paper presents a study of the above notions in categories of polarities; these categories, a generalization of a construct due to Waterman [5], are described as follows. Given a concrete category $\mathbf{K}$ with a functional internal hom $H$ and tensor multiplication $\otimes$, the category $\mathbf{K}_{J}$, of $J$-valued polarities in $\mathbf{K}$, has as objects the triples $P=\left(P_{+}, P_{-}, P_{0}\right)$ where $P_{0}$ is a $\mathbf{K}$-morphism from $P_{+} \otimes P_{-}$into $J$, and as morphisms the analogue of adjoint pairs of maps. Recalling that morphisms $P_{+} \otimes P_{-} \rightarrow J$ correspond to bimorphisms $P_{+} \times P_{-} \rightarrow J$, a polarity morphism from $P$ to $Q$ can roughly be described as a pair of maps $u_{+}: P_{+} \rightarrow Q_{+}$and $u_{-}: Q_{-} \rightarrow P_{-}$such that $\left(u_{+}(a), b\right)$ and $\left(a, u_{-}(b)\right)$ map to the same element of $J$ via the appropriate bimorphisms. An important feature of polarities is that their morphisms are "covariant" in one factor and "contravariant" in the other; this provides each category of polarities with a natural self-duality. If $\mathbf{K}$ has a dualizer, i.e., an object $D$ for which $H(-, D)$ is a self-duality of $\mathbf{K}$, then $\mathbf{K}_{D}$ is isomorphic to the category $\mathbf{K}^{2}$ of arrows in $\mathbf{K}$.

$\mathbf{K}_{J}$ has an underlying set functor which need not be faithful, and so we are not dealing with concrete categories; moreover, examples will show that $\mathbf{K}_{\boldsymbol{J}}$ need not have universal bimorphisms. Nevertheless, $\mathbf{K}_{J}$ has an internal hom functor Hom for which there is an associative and commutative tensor multiplication. This internal hom, which is constructed using the internal hom $H$ in $\mathbf{K}$ and is not, in general, functional, nevertheless does have many of the features of functional internal homs, notably the exponent law $\operatorname{HOM}(P, \operatorname{Hom}(Q, R) \cong$ $\operatorname{Hom}(Q, \operatorname{Hom}(P, R))$ and the first of the two conditions defining functionality. Moreover, there is a dualizer for Hom in $\mathbf{K}_{J}$ iff $\mathbf{K}$ has an object which is free

Received by the editors January 26, 1978 and, in revised form, June 8, 1978. 
on one generator; the latter is a much weaker condition than the existence of a dualizer in $\mathbf{K}$, and so in this respect, Hom is better behaved than $\mathrm{H}$.

Finally, we will see that Proposition 3 of [1], mentioned above, is no longer valid in non-concrete categories; a counterexample is provided by the category Ens $_{J}(J$ any set, Ens the category of sets) where Hom is functional and a functor of universal bimorphisms exists which differs from the tensor multiplication.

Examples of categories of polarities include the category of all bilinear forms (over any field), the category of all residuated maps between complete p.o. sets, and the category of all (finite) matrices with entries from some fixed field.

The first section of the paper recalls a few of the basic facts from [1] about concrete categories with internal hom functors, and provides new information about this setting which is needed in the later sections. In particular, it is proved that if $\otimes$ is a tensor multiplication for $H$ then the natural equivalence $\mathbf{K}(A \otimes B, C) \cong \mathbf{K}(A, H(B, C))$ actually lifts to a $\mathbf{K}$-isomorphism $H(A \otimes$ $B, C) \rightarrow H(A, H(B, C))$, settling a problem left open in [1]. The second section contains the definitions and results on categories of polarities and their internal hom functors, and various examples and counterexamples are presented in section three.

The reader is referred to Banaschewski-Nelson [1] for background on functional internal hom functors, tensor multiplications, etc., and to Mac Lane [3] for relevant material from category theory. The same notation will be used throughout for an object (of any category) and its associated identity map.

The author is grateful to B. Banaschewski for many helpful comments, and to the National Research Council of Canada for financial support.

$\S 1$. Preliminaries. In this section, $\mathbf{K}$ will always be a concrete category, with underlying set functor $|\cdot|$, and with a functional internal hom functor $H$ for which there is a tensor multiplication $\otimes$.

This means that $H: \mathbf{K}^{*} \times \mathbf{K} \rightarrow \mathbf{K}$ is an internal hom functor satisfying:

(F1) For all $A, B \in \mathbf{K}$, there exists $e_{\mathrm{AB}}: A \rightarrow H(H(A, B), B)$ such that $\| e_{\mathrm{AB}}|(a)|(u)=|(u)|(a)$ for all $a \in|A|, u: A \rightarrow B$, and

(F2) For all $A, B, C \in K$, a set map $f:|A| \rightarrow|H(B, C)|$ is $|h|$ for some $h: A \rightarrow H(B, C)$ whenever, for each $b \in|B|,|f()|(b)=\left|h_{b}\right|$ for some $h_{b}: A \rightarrow C$.

In addition, $\otimes: \mathbf{K} \times \mathbf{K} \rightarrow \mathbf{K}$ is a functor for which there are natural isomorphisms $\xi_{A B C}:(A \otimes B, C) \rightarrow(A, H(B, C))$ and universal bimorphisms $\beta_{A B}:|A| \times|B| \rightarrow|A \otimes B|$. The relationship between $\xi$ and $\beta$ can be described as follows: for $f: A \otimes B \rightarrow C, \| \xi_{A B C}(f)|(a)|(b)=|f|\left(\beta_{A B}(a, b)\right)$ for all $a \in|A|$, $b \in|B|$; also, for $f: A \rightarrow C, g: B \rightarrow D$, the map $f \otimes g: A \otimes B \rightarrow C \otimes D$ is uniquely determined by the property that $|f \otimes g| \beta_{A B}=\beta_{C D}|f| \times|g|$. Further, the natural isomorphism $|A| \times|B| \rightarrow|B| \times|A|$ produces a natural isomorphism $A \otimes$ $B \rightarrow B \otimes A$. One other feature of this situation is that $H$ satisfies the exponent 
law, i.e., there are natural isomorphisms $s_{\mathrm{ABC}}: H(A, H(B, C)) \rightarrow$ $H(B, H(A, C))$ such that $\| s_{A B C}|(u)|(b)|(a)=| u|(a)|(b)$.

It was noted in [1, p. 389, Remark 1] that the set map $\xi_{A B C}$ actually lifts to a morphism in $\mathbf{K}$ which is one-one and onto, leaving open the question whether this is actually an isomorphism. The following settles this question affirmatively, and derives some consequences which will be useful later on.

Propostrion 1. For a category $\mathbf{K}$ as above, $\xi_{\mathrm{ABC}}$ lifts to an isomorphism $\psi_{A B C}: H(A \otimes B, C) \rightarrow H(A, H(B, C))$ for all $A, B, C \in \mathbf{K}$.

Proof. Let $f:|A| \times|B| \rightarrow(H(A, H(B, C)), C)$ be the set map with $f(a, b)=$ $\left|e_{\mathrm{BC}}\right|(b)\left|e_{\mathrm{A}, \mathrm{H}(\mathrm{B}, \mathrm{C})}\right|(a)$; then for all $a \in|A|, b \in|B|$,

$$
\begin{aligned}
& f(a,-)=\left|H\left(\left|e_{\mathrm{A}, \mathrm{H}(\mathrm{B}, \mathrm{C})}\right|(a), C\right) e_{\mathrm{BC}}\right| \\
& f(-, b)=\left|H\left(H(A, H(B, C)),\left|e_{\mathrm{BC}}\right|(b)\right) e_{\mathrm{A}, \mathrm{H}(\mathrm{B}, \mathrm{C})}\right|
\end{aligned}
$$

and hence $f$ is a bimorphism. Let $\alpha: A \otimes B \rightarrow H(H(A, H(B, C)), C)$ be the corresponding morphism; then $\alpha$ determines a morphism $\phi: H(A, H(B, C)) \rightarrow$ $H(A \otimes B, C)$ such that $\|\phi|(u)|(x)=\| \alpha|(x)|(u)$ for all $u \in(A, H(B, C))$, all $x \in|A \otimes B|$.

Now, writing $\psi=\psi_{A B C}$, we have, for all $h \in(A \otimes B, C)$,

$$
\begin{aligned}
\| \phi \psi|(h)|\left(\beta_{\mathrm{AB}}(a, b)\right) & =\| \alpha\left|\left(\beta_{\mathrm{AB}}(a, b)\right)\right|(|\psi|(h)) \\
& =\||\psi|(h)|(a)|(b) \\
& =|h|\left(\beta_{\mathrm{AB}}(a, b)\right)
\end{aligned}
$$

which shows that $\phi \psi$ is the identity map on $H(A \otimes B, C)$.

Also, for all $h \in(A, H(B, C))$,

$$
\begin{aligned}
\||\psi \phi|(h)|(a)|(b) & =\| \phi|(h)|\left(\beta_{\mathrm{AB}}(a, b)\right) \\
& =\| \alpha\left|\left(\beta_{\mathrm{AB}}(a, b)\right)\right|(h) \\
& =\| h|(a)|(b)
\end{aligned}
$$

and this shows that $\psi \phi$ is the identity map on $H(A, H(B, C))$, completing the proof.

CoRollary 1. The tensor multiplication in $\mathbf{K}$ is associative, i.e., there is an isomorphism $\gamma_{\mathrm{ABC}}: A \otimes(B \otimes C) \rightarrow(A \otimes B) \otimes C$ such that

$$
\left|\gamma_{A B C}\right| \beta_{A, B \otimes C}\left(|A| \times \beta_{B C}\right)=\beta_{A \otimes B, C}\left(\beta_{A B} \times|C|\right) t
$$

where $t:|A| \times(|B| \times|C|) \rightarrow(|A| \times|B|) \times|C|$ is the obvious set isomorphism. 
Proof. The required homomorphism $\gamma_{A B C}$ is the image of the identity map on $(A \otimes B) \otimes C)$ under the following sequence of natural isomorphisms:

$$
\begin{aligned}
H((A \otimes B) \otimes C,(A \otimes B) \otimes C) & \rightarrow H(A \otimes B, H(C,(A \otimes B) \otimes C)) \\
& \rightarrow H(A, H(B, H(C,(A \otimes B) \otimes C))) \\
& \rightarrow H(A, H(B \otimes C,(A \otimes B) \otimes C)) \\
& \rightarrow H(A \otimes(B \otimes C),(A \otimes B) \otimes C)
\end{aligned}
$$

where the maps in the order they occur, are $\psi_{A \otimes B, C, D}, \psi_{A, B, H(C, D)}, H\left(A, \psi_{B, C, D}^{-1}\right)$ and $\psi_{A, B \otimes C, D}^{-1}$ where $D=(A \otimes B) \otimes C$.

As has been mentioned above, the tensor multiplication is commutative, and thus there are natural isomorphisms $f:(A \otimes B) \otimes C \rightarrow C \otimes(B \otimes A)$ and $g:(C \otimes B) \otimes A \rightarrow A \otimes(B \otimes C)$ such that $g \gamma_{C B A} f \gamma_{A B C}$ and $\gamma_{A B C} g \gamma_{C B A} f$ are both identity maps and this shows that $\gamma_{A B C}$ is an isomorphism.

Corollary 2. The tensor multiplication in $\mathbf{K}$ is internal, i.e. for all $A, B, C \in$ $\mathbf{K}$ there is a map $\mu_{\mathrm{ABC}}: H(A, B) \rightarrow H(A \otimes C, B \otimes C)$ with $\left|\mu_{\mathrm{ABC}}\right|(f)=g \otimes C$.

Proof. Let $\mu_{A B C}=\left|\psi_{H(A, B), A \otimes C, B \otimes C}\right|\left(\left(\alpha_{A B} \otimes C\right) \gamma_{H(A, B), A, C}\right)$, where $\alpha_{A B}$ : $H(A, B) \otimes A \rightarrow B$ is the morphism corresponding to the bimorphism $(A, B) \times|A| \rightarrow|B|$ which maps $(u, a) \leadsto|u|(a)$. Then for $f: A \rightarrow B, a \in|A|$, $c \in|C|$,

$$
\begin{aligned}
\| \mu_{\mathrm{ABC}}|(f)|\left(\beta_{\mathrm{AC}}(a, c)\right) & =\left|\alpha_{\mathrm{AB}} \otimes C\right|\left(| \gamma _ { \mathrm { H } ( \mathrm { A } , \mathrm { B } ) , \mathrm { A } , \mathrm { C } } | \left(\beta_{\mathrm{H}(\mathrm{A}, \mathrm{B}), \mathrm{A} \otimes C}\left(f, \beta_{\mathrm{A}, \mathrm{C}}(a, c)\right)\right.\right. \\
& =\left|\alpha_{\mathrm{AB}} \otimes C\right|\left(\beta_{H(\mathrm{~A}, \mathrm{~B}) \otimes \mathrm{A}, \mathrm{C}}\left(\beta_{\mathrm{H}(\mathrm{A}, \mathrm{B}), \mathrm{A}}(f, a), c\right)\right) \\
& =\beta_{\mathrm{BC}}\left(\left|\alpha_{\mathrm{AB}}\right|\left(\beta_{\mathrm{H}(\mathrm{A}, \mathrm{B}) \mathrm{A}}(f, a)\right), c\right) \\
& =\beta_{\mathrm{BC}}(|f|(a), c)
\end{aligned}
$$

and hence $\mid \mu_{\mathrm{ABC}} \|(f)=f \otimes C$, as required.

The next, and final, item in this section will be used in $\$ 2$, and concerns categories which have an object which is free on one generator. Note that if $\mathbf{K}$ is a category with functional internal hom functor for which there is a dualizer (see $[1, \S 3])$, i.e. an object $D \in \mathbf{K}$ for which all $e_{\mathrm{AD}}: A \rightarrow H(H(A, D), D)$ are isomorphisms, then, as was noted in $[1$, Remark in $\S 3], H(D, D)$ is free on one generator. However, $\mathbf{K}$ having a dualizer is a much stronger hypothesis; the categories of topological spaces and continuous maps, and of p.o. sets and order-preserving maps have no dualizer, but do have objects free on one generator.

Proposition 2. For a category $\mathbf{K}$ as above, which has an object $F$ which is free on one generator $0 \in|F|$, the morphism $\phi_{\mathrm{A}}=\left|e_{\mathrm{FA}}\right|(0): H(F, A) \rightarrow A$ is a natural isomorphism.

Proof. The naturality is clear. 
Let $g: A \rightarrow H(F, A)$ be the morphism corresponding to the morphism $F \rightarrow$ $H(A, A)$ which maps 0 to the identity map on $A$, so that $\| g|(a)|(0)=a$ (see $[1$, Corollary 2 to Proposition 2]).

Then for all $a \in|A|,\left|\phi_{\mathrm{A}} g\right|(a)=\| g|(a)|(0)=a$ and so $\phi_{\mathrm{A}} g$ is the identity map on $A$. Also, for all $u \in(F, A),\left\|g \phi_{A}|(u)|(0)=\right\| g|(|u|(0))|(0)=|u|(0)$; since morphisms on $F$ are completely determined by their effect on 0 , this shows that $g \phi_{A}$ is the identity map on $H(F, A)$ and thus $\phi_{A}$ is an isomorphism.

CoROllary. Under the same hypotheses, the morphism $\nu_{\mathrm{A}}: A \otimes F \rightarrow A$ corresponding to $\phi_{\mathrm{A}}^{-1}: A \rightarrow H(F, A)$ is a natural isomorphism.

Proof. For all $A, B \in \mathbf{K}$ we have the natural equivalences $(A \otimes F, B) \simeq$ $(A, H(F, B)) \simeq(A, B)$, the last coming from the natural isomorphism $\phi_{B}: H(F, B) \rightarrow B$, and hence it follows from the Yoneda lemma (see [3]) that $\nu_{A}$ is a natural isomorphism.

Remark. There is a "converse" to the above corollary: if $\mathbf{K}$ has an object $F$ for which there are natural isomorphisms $A \otimes F \rightarrow A$ for $A \in \mathbf{K}$, then the natural equivalences $(A, B) \simeq(A \otimes F, B) \simeq(A, H(F, B))$ together with the Yoneda lemma (see [3]) imply that $(F,-)$ represents the underlying set functor and hence $\mathbf{K}$ has an object which is free on one generator.

\$2. Categories of polarities. In this section, $\mathbf{K}$ will be a concrete category with a functional internal hom functor $H$, a tensor multiplication $\otimes$ and concrete binary products and equalizers. The latter means that $\mathbf{K}$ has binary products and equalizers, and that the underlying set functor preserves them. Note that if $\mathbf{K}$ has an object which is free on one generator then the underlying set functor is representable and hence if $\mathbf{K}$ has binary products and equalizers they are automatically concrete.

For an object $J$ in $\mathbf{K}$, a $\mathbf{K}$-valued polarity over $J$ is a triple $P=\left(P_{+}, P_{-}, P_{0}\right)$ where $P_{+}, P_{-} \in \mathbf{K}$ and $P_{0}: P_{+} \otimes P_{-} \rightarrow J$. A morphism $P \rightarrow Q$ is a pair $u=$ $\left(u_{+}, u_{-}\right)$, where $u_{+}: P_{+} \rightarrow Q_{+}, u_{-}: Q_{-} \rightarrow P_{-}$, such that the following diagram commutes:

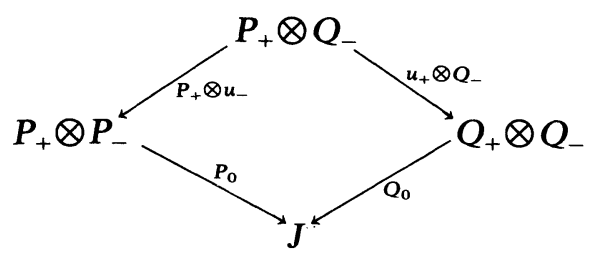

$\mathbf{K}_{J}$ is the category of all $\mathbf{K}$-valued polarities over $J$ and such morphisms. These categories, for $\mathbf{K}$ the category of sets, where $\otimes$ is just cartesian product, were introduced and studied in Waterman [5]. 
For each polarity $P$, let $P_{m}: P_{+} \rightarrow H\left(P_{-}, J\right)$ be the morphism determined by the adjointness $\left(P_{+} \otimes P_{-}, J\right) \cong\left(P_{+}, H\left(P_{-}, J\right)\right)$; then a pair $\left(u_{+}, u_{-}\right) \in$ $\left(P_{+}, Q_{+}\right) \times\left(Q_{-}, J_{-}\right)$is a morphism from $P$ to $Q$ iff $Q_{m} u_{+}=H\left(U_{-}, J\right) P_{m}$. Consequently the category $\mathbf{K}_{J}$ is isomorphic to the comma category ( $T \downarrow S$ ) where $T: \mathbf{K} \rightarrow \mathbf{K}$ is the identity map and $S: \mathbf{K}^{*} \rightarrow \mathbf{K}$ is $H(-, J)$.

For convenience, for the rest of this section, let $H(-, J)=()^{*}$. Note that, as in the proof of Lemma 1 of Banaschewski-Nelson [1], it follows from (F2) for $H$ that there is a morphism $H(A, B) \rightarrow H\left(B^{*}, A^{*}\right)$ mapping $f \leadsto f^{*}$.

In the special case that $J$ is a dualizer in $\mathbf{K},()^{*}$ is a self-duality of $\mathbf{K}$, and then the category $K_{J}$ is equivalent with the category $K^{2}$ of arrows in $K$; some further comments on this are to be found at the beginning of $\$ 3$.

The category $K_{J}$ has an underlying set functor given by $|P|=\left|P_{+}\right|$and $\left|\left(u_{+}, u_{-}\right)\right|=\left|u_{+}\right|$; it is not difficult to see that the underlying set functor is faithful on $(P, Q)$ for all $Q$ iff the map $P_{-} \rightarrow H\left(P_{+}, J\right)$, corresponding to $P_{m}$ by the exponent law for $H$, is a monomorphism.

There is also an "underlying" functor $\mathbf{K}_{J} \rightarrow \mathbf{K}$ given by $P \leadsto P_{+}$, $\left(u_{+}, u_{-}\right) \leadsto u_{+}$; this functor has a left adjoint $F$ defined by: $(F A)_{+}=A,(F A)_{-}=$ $A^{*}$ and $(F A)_{m}=e_{\mathrm{AA}}: A \rightarrow A^{* *}$. This fact, for $\mathbf{K}=\mathbf{E n s}$, is contained in Water$\operatorname{man}[5]$.

Now, to define $\operatorname{HOM}(P, Q)$ for polarities $P, Q \in \mathbf{K}_{J}$ consider the following pullback diagram; where the map $h: H\left(Q_{-}, P_{-}\right) \rightarrow H\left(P_{-}^{*}, Q_{-}^{*}\right)$ takes $f \leadsto f^{*}$ :

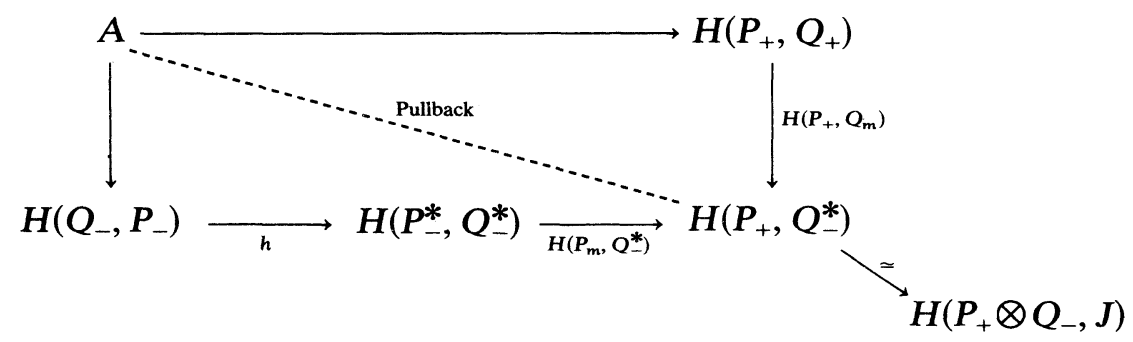

Let $\operatorname{Hom}(P, Q)_{+}=A, \operatorname{Hom}(P, Q)_{-}=P_{+} \otimes Q_{-}$and let $\operatorname{Hom}(P, Q)_{m}$ be the diagonal of the above square followed by the natural isomorphism $\psi_{P_{+} Q_{-} J}^{-1}: H\left(P_{+}, Q_{-}^{*}\right) \rightarrow H\left(P_{+} \otimes Q_{-}, J\right)$. Since $\mathbf{K}$ has concrete binary products and equalizers, the underlying set functor on $\mathbf{K}$ also preserves pullbacks, and so $|\operatorname{Hom}(P, Q)|=\left|\operatorname{Hom}(P, Q)_{+}\right|=(P, Q)$, the set of morphisms from $P$ to $Q$.

Now, to define $\operatorname{Hom}(u, v)$ for morphisms $u=\left(u_{+}, u_{-}\right): S \rightarrow P$ and $v=$ $\left(v_{+}, v_{-}\right): Q \rightarrow R$, consider the following diagram, where the inner and outer squares are the appropriate pullbacks.

Since the right-most and bottom "squares" commute, there is a unique $\mathbf{K}$-morphism $\operatorname{Hom}(P, Q)_{+} \rightarrow \operatorname{Hom}(S, R)_{+}$making the top and left-most "squares" commute. Let $\operatorname{Hom}(u, v)_{+}$be this morphism, and let $\operatorname{Hom}(u, v)_{-}=$ $u_{+} \otimes v_{-}: S_{+} \otimes R_{-} \rightarrow P_{+} \otimes Q_{-}$. It is straightforward to check that these two 


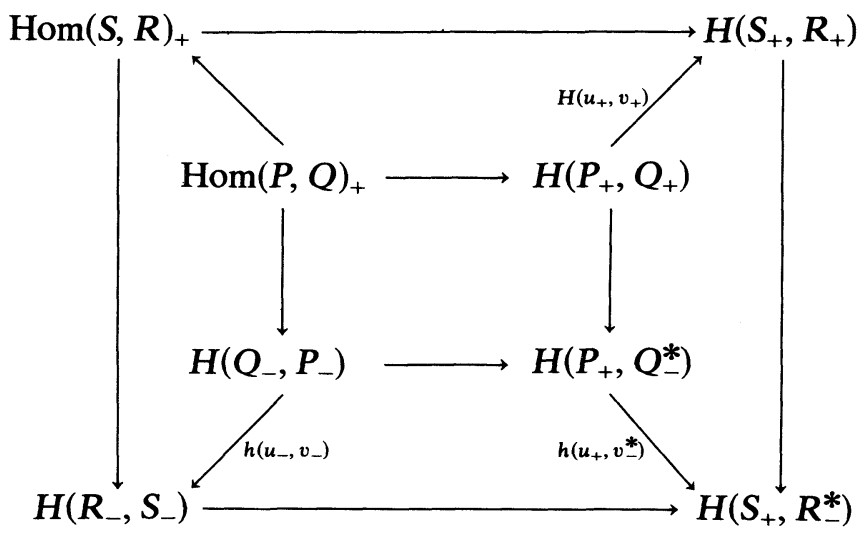

morphisms constitute a $\mathbf{K}_{\mathrm{J}}$-morphism $\operatorname{Hom}(P, Q) \rightarrow \operatorname{Hom}(S, R)$, and that this definition is functorial.

For a functional internal hom $H$, the exponent law is a consequence of the natural equivalence between the bimorphisms $|A| \times|B| \rightarrow|C|$ and the morphisms $A \rightarrow H(B, C)$ (see Banaschewski-Nelson [1, p. 388, Corollary 2]). In varietal categories the exponent law for $H$ is enough to imply its functionality (Linton [2]). Here the situation is different: we will see that Hom is not necessarily functional, but nevertheless satisfies the exponent law, and the latter will be used in showing that there is a tensor multiplication for Hom.

Proposition 3. Hom satisfies the exponent law, i.e., there is a natural isomorphism $\sigma_{P Q R}: \operatorname{Hom}(P, \operatorname{Hom}(Q, R) \rightarrow \operatorname{Hom}(Q, \operatorname{Hom}(P, R)$ such that $\left\|\left|\sigma_{P Q R}\right||(u)||(a)||(b)=\| u|(b) \mid(a)\right.$ for all $u \in(P, \operatorname{Hom}(Q, R))$, all $a \in|Q|$, all $b \in$ $|P|$.

Proof. Consider the following diagram, where the outer square is the pullback defining $\operatorname{Hom}(Q, \operatorname{Hom}(P, R))_{+}$, and the named morphisms are defined below.

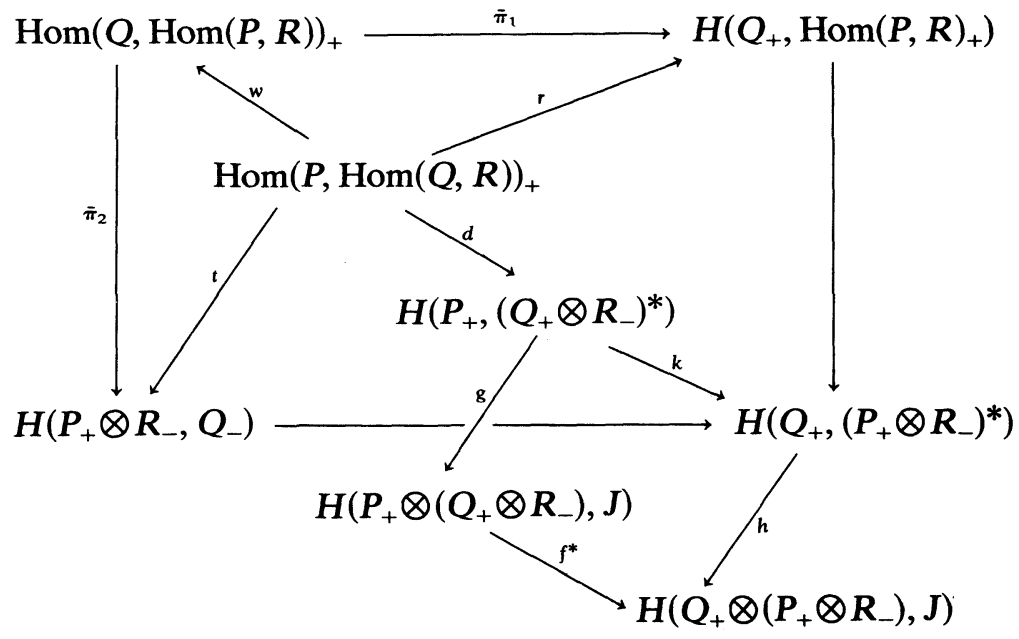


$f: Q_{+} \otimes\left(P_{+} \otimes R_{-}\right) \rightarrow P_{+} \otimes\left(Q_{+} \otimes R_{-}\right)$is the natural isomorphism resulting from the commutativity and associativity of $\otimes$.

$$
\begin{aligned}
& g=\psi_{P_{+}, Q_{+} \otimes R_{-}, J}^{-1}: H\left(P_{+}, H\left(Q_{+} \otimes R_{-}, J\right)\right) \rightarrow H\left(P_{+} \otimes\left(Q_{+} \otimes R_{-}\right), J\right) \\
& h=\psi_{Q_{+}, P_{+} \otimes R_{-} J}^{-}: H\left(Q_{+}, H\left(P_{+} \otimes R_{-}, J\right)\right) \rightarrow H\left(Q_{+} \otimes\left(P_{+} \otimes R_{-}\right), J\right) \\
& k=h^{-1} f^{*} g
\end{aligned}
$$

$d$ is the diagonal of the pullback diagram defining $\operatorname{Hom}(P, \operatorname{Hom}(Q, R))_{+}$, so that $g d=\operatorname{Hom}(P, \operatorname{Hom}(Q, R))_{m}$.

$$
\pi_{1}: \operatorname{Hom}(Q, R)_{+} \rightarrow H\left(Q_{+}, R_{+}\right), \quad \text { and } \quad \pi_{2}: \operatorname{Hom}(Q, R)_{+} \rightarrow H\left(R_{-}, Q_{-}\right),
$$

are the natural projections, and $\bar{\pi}_{1}$ and $\bar{\pi}_{2}$ are the analogous projections on $\operatorname{Hom}(P, \operatorname{Hom}(Q, R))_{+}$.

$$
t=\psi_{P_{+}, R_{-}, Q_{-}}^{-1} H\left(P_{+}, \pi_{2}\right) \bar{\pi}_{1}: \operatorname{Hom}(P, \operatorname{Hom}(Q, R))_{+} \rightarrow H\left(P_{+} \otimes R_{-}, Q_{-}\right) .
$$

Finally, the morphism $r$ is determined by two auxiliary morphisms as follows. Let $r_{1}=s H\left(P_{+}, \pi_{1}\right) \bar{\pi}_{1}: \operatorname{Hom}(P, \operatorname{Hom}(Q, R))_{+} \rightarrow H\left(Q_{+}, H\left(P_{+}, R_{+}\right)\right) \quad$ where $s: H\left(P_{+}, H\left(Q_{+}, R_{+}\right)\right) \rightarrow H\left(Q_{+}, H\left(P_{+}, R_{+}\right)\right)$is the natural isomorphism given by the exponent law for $H$, and let $r_{2}=\psi_{Q_{+}, R_{-}, P_{-}} \bar{\pi}_{2}: \operatorname{Hom}(P, \operatorname{Hom}(Q, R))_{+} \rightarrow$ $H\left(Q_{+}, H\left(R_{-}, P_{-}\right)\right)$. A straightforward computation shows for all $u \in$ $(P, \operatorname{Hom}(Q, R))$, all $a \in\left|Q_{+}\right|$, that $\left(\left\|r_{1}|(u)|(a),\right\| r_{2}|(u)|(a)\right) \in(P, R)$, and hence there is a morphism $r: \operatorname{Hom}(P, \operatorname{Hom}(Q, R))_{+} \rightarrow H\left(Q_{+}, \operatorname{Hom}(P, R)_{+}\right)$with $\| r|(u)|(a)=\left(\left\|r_{1}|(u)|(a),\right\| r_{2}|(u)|(a)\right)$ for all $u \in(P, \operatorname{Hom}(Q, R))$ and all $a \in\left|Q_{+}\right|$.

But then there is a unique morphism . $w: \operatorname{Hom}(P, \operatorname{Hom}(Q, R))_{+} \rightarrow$ $\operatorname{Hom}(Q, \operatorname{Hom}(P, R))_{+}$with $\bar{\pi}_{1} w=r$ and $\bar{\pi}_{2} w=t$.

A straightforward calculation shows that $\sigma_{P Q R}=(w, f)$ is a $\mathbf{K}_{\mathrm{J}}$-morphism from $\operatorname{Hom}(P, \operatorname{Hom}(Q, R))$ to $\operatorname{Hom}(Q, \operatorname{Hom}(P, R))$, and that $\left\|\sigma_{\mathrm{PQR}}|(u)|(a)|(b)=\| u|(b) \mid(a)\right.$. for all $u \in(P, \operatorname{Hom}(Q, R))$, all $a \in|Q|$, all $b \in$ $|P|$. Moreover, further calculation shows that $\left|\sigma_{Q P R} \sigma_{P Q R}\right|$ is the identity map on $(P, \operatorname{Hom}(Q, R))$ and hence the first components of $\sigma_{P Q R}$ and $\sigma_{\mathrm{QPR}}$ are inverse to one another. The second components of these morphisms are inverse to one another by their very definition, and this establishes the fact that $\sigma_{P Q R}$ is an isomorphism, with inverse $\sigma_{\mathrm{QPR}}$. The naturality is clear from the definition of $\sigma_{\mathrm{POR}}$ and this completes the proof.

Corollary 1. Hom satisfies (F1), i.e., there is a morphism $E v_{P Q}: P \rightarrow$ $\operatorname{Hom}(\operatorname{Hom}(P, Q), Q)$ such that $\| E v_{P Q}|(a)|(u)=|u|(a)$ for all $u \in(P, Q)$, all $a \in|P|$.

Proof. $E v_{P Q}$ is the $\mathbf{K}_{J}$-morphism corresponding, via the exponent law for $\operatorname{Hom}$, to the identity map $\operatorname{Hom}(P, Q) \rightarrow \operatorname{Hom}(P, Q)$.

Corollary 2. For all $P, Q, R$ in $\mathbf{K}_{J}$ there is a morphism $\operatorname{Hom}(P, Q) \rightarrow$ $\operatorname{Hom}(\operatorname{Hom}(Q, R), \operatorname{Hom}(P, R))$ mapping $u \leadsto \operatorname{Hom}(u, R)$ for each $u \in(P, Q)$. 
Proof. The required morphism is $\sigma_{P, \operatorname{Hom}(Q, R), R} \operatorname{Hom}\left(P, E v_{\mathrm{QR}}\right)$; a straightforward calculation shows that its first component maps $u$ to $\operatorname{Hom}(u, R)$.

One further comment about the resemblance of Hom to a functional internal hom functor: in a concrete category $\mathbf{K}$ with concrete products the functionality of an internal hom $H$ is equivalent to the existence of suitable embeddings $H(A, B) \rightarrow B^{|A|}$; see [1, Proposition 1]. Now, the category $\mathbf{K}_{J}$ does have products: it is easy to see that the product $P$ of polarities $P_{i}(i \in I)$ has $P_{+}=\prod P_{i+}(i \in I), P_{-}=\amalg P_{i-}(i \in I)$ and $P_{m}$ the unique morphism such that for all $a \in \prod P_{i}, P_{m}(a) e_{j}=P_{j m}(a)$ where $e_{i}: P_{j-} \rightarrow \amalg P_{i-}$ is the natural injection Moreover, it is easy to see that there are naturally arising morphisms $i_{P Q}: \operatorname{Hom}(P, Q) \rightarrow P^{|Q|}$ with $\left|P_{a} i_{P Q}\right|(u)=|u|(a)$ for $a \in|P|, u \in(P, Q)$ where $p_{a}: P_{+}^{|O|} \rightarrow P_{+}$is the $a^{\prime}$ th projection. However, $i_{P Q}$ will not, in general, be an embedding.

The category $\mathbf{K}_{J}$ has a natural self-duality arising from the exponent law for $H$ in $\mathbf{K}$. For a polarity $P$, define $P_{+}^{d}=P_{-}, P_{-}^{d}=P_{+}$and $P_{m}^{d}: P_{-} \rightarrow H\left(P_{+}, J\right)$ to be the map corresponding to $P_{m}$ via the exponent law for $H$. It is obvious that if $u=\left(u_{+}, u_{-}\right) \in(P, Q)$ then $u^{d}=\left(u_{-}, u_{+}\right) \in\left(Q^{d}, P^{d}\right)$, and so this defines a selfduality ( () $)^{d}$ of $\mathbf{K}_{\mathrm{J}}$. Moreover, this is even compatible with Hom in the following sense: for polarities $P$ and $Q$ there is a natural isomorphism $\delta_{\mathrm{PQ}}: \operatorname{Hom}(P, Q) \rightarrow \operatorname{Hom}\left(Q^{d}, P^{d}\right)$ with $\left(\delta_{\mathrm{PQ}}\right)_{+}(u)=u^{d}$ and $\left(\delta_{\mathrm{PQ}}\right)_{-}: Q_{-} \otimes P_{+} \rightarrow$ $P_{+} \otimes Q_{-}$the natural isomorphism given by the commutativity of $\otimes$. This compatibility, together with the exponent law for Hom, produces a tensor multiplication in $\mathbf{K}_{\boldsymbol{J}}$. The next proposition, for $\mathbf{K}$ the category of sets, is also in Waterman [5].

Proposition 4. For all polarities $P, Q$ and $R$ there are natural isomorphisms $\operatorname{Hom}\left(\operatorname{Hom}\left(P, Q^{d}\right)^{d}, R\right) \cong \operatorname{Hom}(P, \operatorname{Hom}(Q, R))$.

Proof. The required isomorphism is the composite of the following sequence of natural isomorphisms: $\operatorname{Hom}\left(\operatorname{Hom}\left(P, Q^{d}\right)^{d}, R\right) \rightarrow \operatorname{Hom}\left(R^{d}, \operatorname{Hom}\left(P, Q^{d}\right)\right) \rightarrow$ $\operatorname{Hom}\left(P, \operatorname{Hom}\left(R^{d}, Q^{d}\right)\right) \rightarrow \operatorname{Hom}(P, \operatorname{Hom}(Q, R))$ where the first and last result from the compatibility of ()$^{d}$ with Hom and the second results from the exponent law for Hom.

The tensor multiplication $\operatorname{Hom}\left(-,(-)^{d}\right)^{d}$ is clearly commutative, and its associativity results from this, and the following sequence of natural isomorphisms: $\operatorname{Hom}\left(\operatorname{Hom}\left(P, Q^{d}\right)^{d}, R^{d}\right)^{d} \cong \operatorname{HOm}\left(R, \operatorname{Hom}\left(P, Q^{d}\right)\right)^{d} \simeq$ $\operatorname{Hom}\left(P, \operatorname{Hom}\left(R, Q^{d}\right)\right)^{d} \cong \operatorname{Hom}\left(\operatorname{Hom}\left(Q, R^{d}\right)^{d}, P^{d}\right)^{d}$. For $\mathbf{K}$ the category of sets this associativity is proved by direct computation in Waterman [5].

For each polarity $\Delta \in \mathbf{K}_{J}$ the exponent law for $\operatorname{Hom}$ implies that $\operatorname{Hom}(-, \Delta)$ is self-adjoint on the right; one question that arises is whether the self-duality ()$^{d}$ of $\mathbf{K}_{J}$ is equivalent to some $\operatorname{Hom}(-, \Delta)$, and whether $\mathbf{K}$ has a dualizer. 
These questions have a surprisingly tidy answer:

Proposition 5. There is a polarity $\Delta$ for which there is a natural isomorphism $\operatorname{Hom}(-, \Delta) \simeq()^{d}$ iff $\mathbf{K}$ has an object which is free on one generator.

Proof. Suppose there is a natural isomorphism $\operatorname{Hom}(-, \Delta) \simeq()^{d}$. For each $A \in K$, let $F A$ be the polarity with $(F A)_{+}=A,(F A)_{-}=H(A, J)$ and $(F A)_{m}=$ $e_{\mathrm{AA}}$. Then there is a natural isomorphism $A=(F A)_{-}^{d} \rightarrow \operatorname{Hom}(F A, \Delta)=A \otimes \Delta_{-}$ and by the remark at the end of $\S 1$, this ensures that $\mathbf{K}$ has an object which is free on one generator.

For the converse, suppose $F \in \mathbf{K}$ is free on one generator $0 \in|F|$ and let $\Delta$ be the polarity with $\Delta_{+}=J, \Delta_{-}=F$ and $\Delta_{m}: J \rightarrow H(F, J)$ the natural isomorphism (in the notation of Proposition 2, $\Delta_{m}=\phi_{J}^{-1}$ ).

For $P \in \mathbf{K}_{J}$ let $u_{1}=\phi_{p-} \pi_{2}: \operatorname{Hom}(P, \Delta)_{+} \rightarrow P_{-} \quad\left(\right.$ where $\pi_{2}: \operatorname{Hom}(P, \Delta)_{+} \rightarrow$ $H\left(\Delta_{-}, P_{-}\right)$is the second projection) and let $u_{2}=\nu_{P_{+}}^{-1}: P_{+} \rightarrow P_{+} \otimes F$ be the natural isomorphism. We will see that $u_{P}=\left(u_{1}, u_{2}\right)$ is a natural isomorphism from $\operatorname{Hom}(P, \Delta)$ to $P^{d}$. Since the naturality of $u_{P}$ follows from that of $\phi$ and $\nu$ and since $u_{2}$ is by definition an isomorphism, it is only necessary to show that $u_{1}$ is an isomorphism, and that $P_{m}^{d} u_{1}=u_{2}^{*} \operatorname{Hom}(P, \Delta)_{m}$. Verification of the latter is a straightforward computation; for the former, let $r: P_{-} \rightarrow H\left(P_{+}, J\right) \times$ $H\left(F, P_{-}\right)$be the map with $\tilde{\pi}_{1} r=P_{m}^{d}, \tilde{\pi}_{2} r=\phi_{P_{-}}^{-1}$ where $\tilde{\pi}_{1}$ and $\tilde{\pi}_{2}$ are the projections on $H\left(P_{+}, J\right) \times H\left(F, P_{-}\right)$. Then $r$ factors through $\operatorname{Hom}(P, \Delta)_{+}$to provide a map which is the inverse of $u_{1}$, and this completes the proof.

As was mentioned before, if $\mathbf{K}$ has a dualizer then it has an object which is free on one generator, but the latter is a strictly weaker assumption.

Corollary 1. If $F \in \mathbf{K}$ is free on one generator then $\Delta=\left(J, F, \nu_{F}\right)$ is a dualizer in $\mathbf{K}_{\mathrm{J}}$.

Proof. The proof of the proposition provides natural isomorphisms $u_{\mathrm{P}}: \operatorname{Hom}(P, \Delta) \rightarrow P^{d}$ for $P \in \mathbf{K}_{\mathrm{J}}$, and it is straightforward to check that

$$
P \stackrel{E v_{P} \Delta}{\longrightarrow} \operatorname{Hom}(\operatorname{Hom}(P, \Delta), \Delta) \stackrel{{ }^{u} \operatorname{Hom}(P, \Delta)}{\longrightarrow} \operatorname{Hom}(P, \Delta)^{d} \stackrel{\left(u_{\mathrm{P}}{ }^{-1}\right)^{d}}{\longrightarrow}\left(P^{d}\right)^{d}=P
$$

is the identity morphism on $P$. Since $u_{\operatorname{Hom}(P, \Delta)}$ and $\left(u_{P}^{-1}\right)^{d}$ are isomorphisms, it follows that $E v_{P \Delta}$ is an isomorphism.

This brings us to the question of universal bimorphisms in $\mathbf{K}_{J}$. For all polarities $P, Q, \beta_{P_{+} Q_{+}}:|P| \times|Q| \rightarrow\left|P_{+} \otimes Q_{+}\right|=\left|\operatorname{Hom}\left(P, Q^{d}\right)^{d}\right|$ is actually a bimorphism (relative to $\mathbf{K}_{J}$ ). Moreover, if Hom is functional (i.e. satisfies (F2)) then every bimorphism $\phi:|P| \times|Q| \rightarrow|R|$ factors through $\beta_{P_{+} Q_{+}}$: functionality implies that there is a morphism $\bar{\phi}: P \rightarrow \operatorname{Hom}(Q, R)$ with $\| \bar{\phi}|(p)|(q)=\phi(p, q)$ and then the fact that $\operatorname{Hom}\left(-,(-)^{d}\right)^{d}$ is a tensor multiplication yields a 
morphism $u: \operatorname{Hom}\left(P, Q^{d}\right)^{d} \rightarrow R$ with $|u| \beta_{P_{+} Q_{+}}=\phi$. The only obstruction to $\beta$ being universal is that this $u$ may not be uniquely determined, if the underlying set functor is not faithful on $\left(\operatorname{Hom}\left(P, Q^{d}\right)^{d}, R\right)$. Using the following lemma, examples are given in the next section to show that $K_{\mathrm{J}}$ need not have universal bimorphisms.

LEMMA. If $\mathbf{K}$ has an object $F$ which is free on one generator, then there is a universal bimorphism on $|P| \times|\bar{F}|$ relative to $\mathbf{K}_{J}$ (where $\bar{F}_{+}=F, \bar{F}=J$ and $\bar{F}_{M}: F \rightarrow H(J, J)$ maps the generator of $F$ to the identity map on $\left.J\right)$ iff $P_{m}^{d}=g h$ where $g$ is a monomorphism and $h$ is a right invertible map in $\mathbf{K}$.

Proof. Suppose $\beta:|P| \times|\bar{F}| \rightarrow|Q|$ is a universal bimorphism. The map $\phi:|P| \times|\bar{F}| \rightarrow|P|$ such that $\phi(a, b)$ is the image of $b$ under the map $F \rightarrow P$ mapping the free generator 0 to $a$ is a bimorphism, and hence there exists a unique morphism $u: Q \rightarrow P$ with $|u| \beta=\phi$. Let $v: P \rightarrow Q$ be a morphism with $|v|=\beta(-, 0)$.

Then for all $a \in\left|P_{+}\right|,|v u|(\beta(a, 0))=|v|(\phi(a, 0))=|v|(a)=\beta(a, 0)$ and thus $|v u| \beta=\beta$. The universality of $\beta$ then implies that $v u$ is the identity morphism on $Q$, and so $v_{+} u_{+}$and $u_{-} v_{-}$are identity maps. Moreover, for all $a \in\left|P_{+}\right|$, $\left|u_{+} v_{+}\right|(a)=\left|u_{+}\right|(\beta(a, 0))=a$ and this shows that $u_{+} v_{+}$is the identity map on $P_{+}$. Since $\left(u_{-}, u_{+}\right)$is a morphism from $P^{d}$ to $Q^{d}$ and $u_{+}$is an isomorphism with inverse $v_{+}$it follows that $P_{m}=H\left(v_{+}, J\right) Q_{m}^{d} u_{-}$. Now $Q_{m}^{d}$ is a monomorphism because the universality of $\beta$ implies that the underlying set functor is faithful on $(Q, R)$ for all $R$, and so $H\left(v_{+}, J\right) Q_{m}^{d}$ is a monomorphism; since $u_{-}$has a right inverse, this produces the required factorization.

Conversely, suppose $P_{m}^{d}=g h$ where $h: P_{-} \rightarrow B$ has right inverse $f$ and $g: B \rightarrow H\left(P_{+}, J\right)$ is a monomorphism. Let $Q_{+}=P_{+}, Q_{-}=B$ and $Q_{m}^{d}=g$; then $\beta:|P| \times|\bar{F}| \rightarrow|Q|$ defined by: $\beta(a, b)$ is the image of $b$ under the map $F \rightarrow P_{+}$ mapping 0 to $a$, can be shown to be a bimorphism. We will see that it is universal. Suppose $\phi:|P| \times|F| \rightarrow|R|$ is any bimorphism, and let $u=$ $\left(u_{+}, u_{-}\right): P \rightarrow R$ with $|u|=\phi(-, 0)$. Then since $\left(P_{+}, h\right)$ is a morphism from $Q$ to $P,\left(u_{+}, u_{-} h\right)$ is a morphism from $Q \rightarrow R$, and moreover $\left|u_{+}\right| \beta=\phi$. The uniqueness of $\left(u_{+}, u_{-} h\right)$ follows from the fact that $Q_{m}^{d}=g$ is mono and hence the underlying set functor is faithful on $(Q, R)$.

\section{§3. Examples and counterexamples.}

1. $\mathbf{K}_{J}$ when $J$ is a dualizer in $\mathbf{K}$.

It has already been mentioned that if $J$ is a dualizer in $K$ then $K_{J} \simeq K^{2}$, the category of arrows in $\mathbf{K}$. Here, a morphism $u=\left(u_{+}, u_{-}\right): f \rightarrow g$ is a pair of maps such that $g u_{+}=u_{-} f$, and $\operatorname{Hom}(f, g)$ is just the diagonal of the following pullback diagram (where $D$ and $C$ indicate domain and co-domain respectively). 


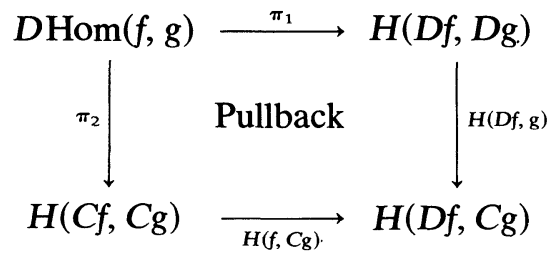

Note that if $g$ is an isomorphism then $\operatorname{Hom}(f, g) \cong H(f, C g)$ with equality holding if $g$ is an identity map. Since $J$ is a dualizer, $H(J, J)$ is free on one generator in $\mathbf{K}$, and so $\mathbf{K}^{2}$ has a dualizer; in fact the dualizer in $K^{2}$ is just the identity map on $J$, and the functor ()$^{d}$ corresponds to $H(-, J)$.

The internal hom just described can of course be defined in any category $\mathbf{K}^{2}$, irregardless of whether or not $\mathbf{K}$ has a dualizer, and the resulting functor will satisfy the exponent law and (F1). However, without the assumption of a dualizer in $\mathbf{K}, \mathbf{K}^{2}$ need $\mathrm{n}^{+}$have a dualizer. In fact, there need not even be a tensor multiplication for Hom; this will be shown for $\mathbf{K}=\mathbf{E n s}$, the category of sets, in the next part of this section.

There is a second internal hom for $\mathbf{K}^{\mathbf{2}}$ which is described as follows: for $\mathbf{K}$-morphisms $f$ and $g, \operatorname{Hom}(f, g)$ is the left vertical arrow (i.e. the second projection) in the above pullback diagram defining $\operatorname{Hom}(f, g)$. There is always a tensor multiplication for Hom, which is the functor $\mathbf{K}^{2} \times \mathbf{K}^{2} \rightarrow \mathbf{K}^{2}$ obtained by defining the tensor multiplication $\otimes$ in $\mathbf{K}$ "pointwise". For a cartesian closed category $\mathbf{K}, \mathrm{HOM}$ is precisely the functor of exponentiation in $\mathbf{K}^{\mathbf{2}}$; these facts can be found in Nelson [4]. HOM also satisfies the exponent law and (F1) but it can be shown that it is not functional even in the case that $\mathbf{K}$ is the category of sets. Moreover, even if $\mathbf{K}$ has a dualizer, there need not be a dualizer for HOM; the category CJSL of complete join semilattices will be seen to provide such an example.

2. Ens. the category Ens of sets obviously has an internal hom functor; here the tensor multiplication is just cartesian product.

For any set $\boldsymbol{J}$, the functor Hom in $\mathbf{E n s}_{\boldsymbol{J}}$ is actually functional, i.e., satisfies (F2): If $v: P_{+} \rightarrow \operatorname{Hom}(Q, R)_{+}$is a set map for which there are morphisms $u_{b}=\left(u_{b_{+}}, u_{b_{-}}\right): P \rightarrow R$ such that $|v()|(b)=u_{b_{+}}$for each $b \in Q_{+}$then define $w: Q_{+} \times R_{-} \rightarrow P_{-}$by $w(b, c)=u_{b_{-}}(c)$; a straightforward calculation shows that $(v, w)$ is a morphism from $P$ to $\operatorname{Hom}(Q, R)$.

A similar argument shows that in Ens ${ }^{2}$, Hom is functional, although the functor HOM is not functional even here. The. following discussion shows that there is no tensor multiplication for Hom in Ens ${ }^{2}$. Suppose there were such a tensor multiplication $\otimes$ and let $f=\overline{0} \otimes \overline{0}: A \rightarrow B,(\overline{0}: 2 \rightarrow 2$ the constant map with value 0$)$. Then for any $h: 1 \rightarrow 2, \operatorname{Hom}(\overline{0}, h)$ is a constant map $2 \rightarrow 4$, and from this one can see that there are $2^{4}$ morphisms $\overline{0} \rightarrow \operatorname{Hom}(\overline{0}, h)$. Also, there are $2^{|B-i m f|}$ morphisms $f \rightarrow h$, and thus $|B-\operatorname{imf}|=4$. Now let $g: 3 \rightarrow 2$ be defined by $g(0)=0, g(1)=g(2)=1$. Then straightforward calculations show that there 
are 10 morphisms $\overline{0} \rightarrow g$, and that $\operatorname{Hom}(\overline{0}, g)$ is isomorphic to the map $10 \rightarrow 4$ which takes 0 and 1 to 0 and everything else to 1 . From this one can show that there are $2^{2} \cdot 2^{2}$ morphisms $(u, v): \overline{0} \rightarrow \operatorname{Hom}(\overline{0}, g)$ with $u(0)=0$ and $2^{2} \cdot 7^{2}$ morphisms $(u, v): \overline{0} \rightarrow \operatorname{Hom}(\overline{0}, g)$ with $u(0)=1$, and so there are $2^{2} \times 53$ morphisms $\overline{0} \rightarrow \operatorname{Hom}(\overline{0}, g)$ all together. On the other hand, since $|B-\operatorname{imf}|=4$, it follows that for each $\alpha: 2 \rightarrow 3$ which is the first half of a morphism $f \rightarrow g$ there are exactly $2^{4}$ morphisms $\beta: B \rightarrow 2$ with $(\alpha, \beta)$ a morphism $f \rightarrow g$. Consequently the number of morphisms $f \rightarrow g$ is divisible by $2^{4}$. Since $2^{2} \times 53$ evidently does not have this property this shows that there is no tensor product $\overline{0} \otimes \overline{0}$ relative to Hom.

Since the one-element set is free on one generator in Ens, the polarity $\left(J, 1, \pi_{1}\right)$ is a dualizer for Hom in Ens $\mathbf{E}_{J}$ for any set $J$. Moreover, Ens , has universal bimorphisms: for polarities $P$ and $Q$, write $\operatorname{Hom}\left(P, Q^{d}\right)_{m}=g h$ where $g$ is a monomorphism and $h$ is epi, and hence right invertible. Then the polarity $U$ with $U_{+}=\operatorname{Hom}\left(P, Q^{d}\right)_{+}^{d}\left(=P_{+} \times Q_{+}\right), U_{-}=D g$ (the domain of $g$ ) and $U_{m}^{d}=g$ is a retract of $\operatorname{Hom}\left(P, Q^{d}\right)^{d}$ with the property that the underlying set functor is faithful on $(U, R)$ for all $R$. But now every bimorphism $|P| \times|Q| \rightarrow$ $|R|$ which factors through $\operatorname{Hom}\left(P, Q^{d}\right)^{d}$ also factors through $U$, and the latter factorization will be unique. Since Hom is functional, the discussion immediately following Corollary 1 of Proposition 5 shows that the identity map $P_{+} \times Q_{+} \rightarrow P_{+} \times Q_{+}$is a universal bimorphism with respect to Ens J Thus Ens $_{J}$ has a functor of universal bimorphisms, and if card $J \geq 2$, this differs from the tensor product in $\mathbf{E n s}_{\mathrm{J}}$, despite the fact that here Hom is functional. This then provides the promised counterexample to [1, Proposition 3] if the "concreteness" restriction is relaxed.

3. POEns. The category POEns of partially ordered sets and orderpreserving maps has a functional internal hom functor, and here, as in Ens, the tensor product is just cartesian product, and the one-element set is free on one generator, so that there is a dualizer in POEns.

Unlike the situation for $\mathbf{E n s}_{J}$, there are not universal bimorphisms in POEns $_{2}$, for 2 the two-element chain: Let $P_{+}=\mathbf{2}$, then $H\left(P_{+}, 2\right)$ is the three element chain. Let $P_{-}$be the disjoint union of two two-chains, and let $P_{m}^{d}: P_{-} \rightarrow H\left(P_{+}, 2\right)$ be a function mapping onto $H\left(P_{+}, 2\right)$, Then $P_{m}^{d}$ does not decompose into a right invertible map followed by a monomorphism, and so the lemma at the end of $\S 2$ shows that POEns, does not have universal bimorphisms.

Moreover, the functor Hom in POEns 2 is not functional: Let $P=\left(\mathbf{2}, \mathbf{2}, P_{\mathbf{0}}\right)$ where $P_{0}: \mathbf{2} \times \mathbf{2} \rightarrow \mathbf{2}$ is the constant map with value 0 . Then $\operatorname{Hom}(P, P)_{+}=$ $\{\overline{0}, \overline{1}, i\}^{2}$. Let $f:\left|\operatorname{Hom}(P, P)_{+}\right| \rightarrow\left|\operatorname{Hom}(P, P)_{+}\right|$be the set map with $f(i, u)=$ $(i, \overline{0}), f(\overline{0}, u)=(\overline{0}, i)$ and $f(\overline{1}, u)=(\overline{1}, \overline{1})$ for all $u \in\{\overline{0}, \overline{1}, i\}$, then $f$ satisfies the hypotheses of F2 but does not underly a morphism in POEns 2 since it is not even order-preserving. 
4. CJSL. The category CJSL of complete join semilattices and complete join-preserving maps has a functional internal hom functor, and the twoelement chain $\mathbf{2}$ is a dualizer, so that $\mathbf{C J S L}^{2} \simeq \mathbf{C J S L}_{2}$. Here, as in POEns 2 , the functor Hom is not functional: $D \operatorname{Hom}(\overline{0}, \overline{0})=\{\overline{0}, i\} \times\{\overline{0}, i\}$ and the set map $f: D \operatorname{Hom}(\overline{0}, \overline{0}) 2 \rightarrow D \operatorname{Hom}(\overline{0}, \overline{0})$ with $f(\overline{0}, u)=(\overline{0}, i)$ and $f(i, u)=(i, \overline{0})$ for each $u$, satisfies the hypotheses of F2 but does not underly a morphism $\operatorname{Hom}(\overline{0}, \overline{0}) \rightarrow$ $\operatorname{Hom}(\overline{0}, \overline{0})$.

Moreover, as was mentioned above, despite the fact that CJSL has a dualizer, there is no dualizer for HOM in $\mathbf{C J S L}^{2}$ : Suppose there were a dualizer $\Delta$ for HOM, then for each morphism $f$ in CJSL, $e_{C f}: C f \rightarrow$ $H(H(C f, C \Delta), C \Delta)$ would be an isomorphism so that $C \Delta$ would be a dualizer in CJSL. Thus $C \Delta=\mathbf{2}$. Now let $f$ be the identity map on $\mathbf{2}$, then since $\mathbf{2}$ is free in CJSL on one generator, it follows that $\operatorname{Hom}(f, \Delta) \simeq H(f, \Delta) \simeq \Delta$. Thus $\operatorname{HOM}(\operatorname{HOM}(f, \Delta), \Delta) \simeq \operatorname{HOM}(\Delta, \Delta)$. Now it is easy to see that if card $D \Delta \geq 3$ then card $D \operatorname{HOM}(\Delta, \Delta) \geq 3$, and this shows that card $D \Delta \leq 2$. If $D \Delta=1$ then $\operatorname{DHOM}(\Delta, \Delta)=1$. If $D \Delta=2$ and $\Delta$ is constant then card $D \operatorname{HOM}(\Delta, \Delta)=4$. Thus $D \Delta=\mathbf{2}$ and $\Delta$ is the identity map on 2 . But then for all $g \in \mathbf{C J S L}^{2}$, $\operatorname{HOM}(g, \Delta)$ is an isomorphism, and so this shows that $\Delta$ is not a dualizer.

Finally, we see that CJSL $^{2}$ does not have universal bimorphisms. Let $A=(\omega \cup\{\omega\}) \times 2$ and let $B$ be the subset of $A$ consisting of all points except $(\omega, 0)$ with the induced order; then the mapping $A \rightarrow B$ which takes $(\omega, 0)$ to $(\omega, 1)$ and maps everything else identically does not decompose into a monomorphism preceded by a right invertible map, and so by the lemma at the end of $\$ 2, \mathbf{C J S L}^{2}$ does not have universal bimorphisms.

5. The final example is the category $\mathbf{M}$ whose objects are all (finite) matrices with entries from some field, where a map $A \rightarrow B$ is a pair $(C, D)$ of matrices with $D A=B C$. Clearly $\mathbf{M}=\mathbf{M}_{0}^{2}$, where $\mathbf{M}_{0}$ is the category whose morphisms are all matrices, with composition given by matrix multiplication, and whose objects are the identity matrices. Some preliminary discussion is needed to describe the internal hom and tensor multiplication in $\mathbf{M}_{0}$.

Recall that the category $\mathbf{V}_{f d}$ of all finite-dimensional vector spaces has a functional internal hom $\boldsymbol{H}$ for which there is a tensor multiplication and a dualizer. For vector spaces $U$ and $V$, bases $\left\{u_{1}, \ldots, u_{m}\right\}$ and $\left\{v_{1}, \ldots, v_{n}\right\}$ for $U$ and $V$ respectively, give rise to a basis $\left\{\delta_{11}, \delta_{12}, \ldots, \delta_{1 m}, \delta_{21}, \ldots\right.$, $\left.\delta_{2 m}, \ldots, \delta_{n m}\right\}$ for $H(U, V) ; \delta_{i j}$ is the linear transformation mapping $u_{j}$ to $v_{i}$ and all other basis vectors to 0 . Each choice of bases for $U$ and $V$ produces the familiar correspondence between linear transformations from $U$ to $V$ and $n \times m$ matrices; the matrix of a linear transformation $f: U \rightarrow V$ has the components of $f\left(u_{j}\right)$ as the $j$ th column. Given $f: R \rightarrow U$ and $g: V \rightarrow S$, and a particular choice of bases for $U, V, R$ and $S$, easy calculations show that the matrix corresponding to the map $H(f, g): H(U, V) \rightarrow H(R, S)$, relative to the bases for $H(U, V)$ and $H(R, S)$ as described above, is $A^{t} \otimes B$, where $A$ 
and $B$ are the matrices corresponding to $f$ and $g$ respectively, ()$^{t}$ indicates transpose, and $\otimes$ is the Kronecker product of matrices, so that

$$
A \otimes B=\left[\begin{array}{cccc}
A b_{11} & A b_{12} & \ldots & A b_{1 n} \\
A b_{21} & A b_{22} & \ldots & A b_{2 n} \\
\cdot & \cdot & & \cdot \\
\cdot & \cdot & & \cdot \\
\cdot & \cdot & & \cdot \\
A \dot{b}_{k 1} & A \dot{b}_{k 2} & \ldots & A b_{k n}
\end{array}\right]
$$

Moreover, the matrix corresponding to the map $f \otimes g$ is just $A \otimes B$.

$\mathbf{M}_{\mathbf{0}}$ is essentially the skeleton subcategory of $\mathbf{V}_{f d}$ consisting of the spaces $k^{n}$ ( $k$ the field). More precisely, $\mathbf{M}_{0}$ is the category whose morphisms are all finite matrices with entries from $k$, composition is just matrix multiplication, and the objects are the identity matrices $I_{n} . \mathbf{M}_{0}$ has an underlying set functor: the underlying set of $I_{n}$ is the set $k^{n}$ and the underlying morphism of an $m \times n$ matrix $A$ is given by $|A|: k^{n} \rightarrow k^{m}$,

$$
|A|(x)=A\left[\begin{array}{c}
x_{1} \\
\cdot \\
\cdot \\
\cdot \\
x_{n}
\end{array}\right]
$$

There is a canonical isomorphism from the set of all $m \times n$ matrices (= the set of all morphisms $\left.I_{n} \rightarrow I_{m}\right)$ to $\left|I_{m n}\right|=k^{m n}$ given by

$$
\tilde{A} \leadsto\left(a_{11}, a_{12}, \ldots, a_{1 n}, a_{21}, a_{22}, \ldots, a_{2 n}, \ldots, a_{m 1}, \ldots, a_{m n}\right) \text {. }
$$

Define $H: \mathbf{M}_{0}^{*} \times \mathbf{M}_{0} \rightarrow \mathbf{M}_{0}$ by $H\left(I_{n}, I_{m}\right)=I_{n m}$ and $H(A, B)=A^{t} \otimes B$; the functionality of $H$ follows from the matrix identity $(A \otimes B)(C \otimes D)=A C \otimes B D$. Moreover, an easy calculation shows, for $A: I_{n} \rightarrow I_{m}, B: I_{k} \rightarrow I_{n}, C: I_{m} \rightarrow I_{k}$, that $|H(A, B)|(\tilde{C})=(B C A)$. and thus $H$ is an internal hom functor for $\mathbf{M}_{0}$. The Kronecker product provides a tensor multiplication, and $\mathbf{M}_{0}$ inherits the property from $\mathbf{V}_{f d}$ of having universal bimorphisms, so $H$ is functional. The identity matrix $I_{1}=(1)$ is a dualizer for $H$.

Consequently the category $\mathbf{M}=\mathbf{M}_{0}^{2}$ whose objects are all matrices has an internal hom functor Hom for which there is a dualizer and a tensor multiplication. It can be shown that Hom is not functional.

A detailed description of Hom can be given by first observing what pullbacks in $\mathbf{M}_{0}$ look like. The product, in $\mathbf{M}_{0}$, of $I_{n}$ and $I_{m}$ is $I_{n m}$ with projection maps $\pi_{1}=\left(I_{n}, 0\right)$ and $\pi_{2}=\left(0, I_{m}\right)$ where the first 0 is the $n \times m$ zero matrix and the second 0 is the $m \times n$ zero matrix. The pullback of $A: I_{n} \rightarrow I_{m}$ and $B: I_{k} \rightarrow I_{m}$ is the equalizer of the maps $(A, 0): I_{n k} \rightarrow I_{m}$ and $(0, B): I_{n k} \rightarrow I_{m}$, and this is just the kernel of $(A,-B)$. Finally, the kernel, $\operatorname{ker} A$, an $m \times n$ matrix of rank $k$ 
is the matrix consisting of the last $n-k$ columns of the matrix which results from performing Gramm-Schmidt orthogonalization on the columns of the matrix $\left(A^{t}, I_{n}\right)$.

A straightforward calculation now shows that for matrices $A$ and $B,(m \times n$ and $k \times h$ respectively), $\operatorname{Hom}(A, B)=\left(I_{n} \otimes B, 0\right) \operatorname{Ker}\left(A^{t} \otimes I_{k},-B \otimes I_{n}\right)$.

\section{REFERENCES}

1. B. Banaschewski and E. Nelson. Tensor products and bimorphisms. Can. Math. Bull. 19 (1976), 385-402.

2. F. E. J. Linton, Autonomous equational categories, J. Math. Mech. 15 (1966), 637-642.

3. S. Mac Lane, Categories for the Working Mathematician, Springer 1971.

4. E. Nelson, On Exponentiating Exponentiation. Manuscript, McMaster University, 1978.

5. A. G. Waterman, General-Valued Polarities, Ph.D. Thesis, Harvard University, 1971.

Department OF MATHEMATiCS

MCMASTER UNIVERSTTY

HAMILTON, ONT. L8S 4K1 